\title{
Indovina chi viene a cena? \\ Questioni psico-socio-giuridiche \\ nel ciclo di vita della coppia mista
}

\author{
di Maria Garro*, Alessandra Salerno ${ }^{* *}$ e Daniela Sciortino ${ }^{* * *}$
}

\section{Introduzione}

Mutamenti sociali, mobilità transnazionale e valorizzazione del benessere dell'individuo hanno caratterizzato la società moderna e contribuito alla formazione delle coppie miste; un fenomeno che coinvolge individui che provengono da paesi a forte pressione migratoria, come l'Arcipelago delle Filippine, la Romania, la Polonia o l'Albania da cui giungono prevalentemente le donne, mentre l'Africa è, in genere, il paese di origine degli uomini che sposano un'italiana (senegalesi, tunisini, marocchini) (Carrà, 2011). L'analisi delle caratteristiche della coppia mista consente di esplorare la praticabilità dell'integrazione di differenti esperienze culturali sul piano dei rapporti di coppia, familiari e sociali (Scabini, Regalia e Giuliani, 2007), esprimendo il livello più profondo d'incontro tra culture e identità differenti che sembra ormai costituire la base della società moderna e che necessita approfondimento, studio e attenzione scientifica crescenti.

\section{Il ciclo vitale della coppia mista}

Le coppie miste presentano in maniera più accentuata i problemi evolutivi che, solitamente, ogni coppia si trova ad affrontare. La prima fase del ciclo di vita familiare, ovvero la formazione della coppia, prende avvio unipa.it

* Dipartimento di Psicologia, Università degli Studi di Palermo, e-mail: maria.garro@

${ }^{* *}$ Dipartimento di Psicologia, Università degli Studi di Palermo, e-mail: alessandra. salerno@unipa.it

${ }^{* * *}$ Psicologa, esperta in valutazione psicologica, e-mail: danielasciortino@libero.it

Psicologia di comunità, n. 1/2013 
dall'uscita del giovane adulto dalla propria famiglia d'origine. Nel caso delle coppie miste, la reazione delle famiglie alla decisione del figlio di intraprendere una relazione con un membro di una diversa nazionalità è inizialmente di sorpresa, tramutandosi in "shock" quando le differenze con il partner sono molto evidenti o quando esistono differenze alle quali la famiglia di origine attribuisce un'importanza fondamentale (Gozzoli e Regalia, 2005; Salerno, 2010). Una delle preoccupazioni più comuni è che il figlio possa perdere il contatto con la cultura di origine, che la sua identità e quella della famiglia possano essere "contaminate" dal contatto con un mondo diverso dal proprio.

Alcune culture da sempre hanno adottato sistemi prescrittivi che indicano con precisione chi idealmente si deve scegliere, o evitare di scegliere, come proprio partner, restringendo così le scelte individuali. Ecco perché senso di colpa e di solitudine sembrano accomunare i membri delle coppie miste, soprattutto se appartenenti a culture dove l'approvazione dei familiari è considerata fondamentale. Tali sentimenti negativi vengono attenuati dall'esercizio dei modelli familiari tradizionali all'interno della vita coniugale (Gozzoli e Regalia, 2005). Sul versante opposto, alcuni individui cercano di gestire il senso di colpa provato nei confronti della famiglia d'origine allontanandosi totalmente dal proprio patrimonio culturale, in nome della libertà di scelta che ogni individuo deve poter avere sulla propria vita.

Al periodo iniziale caratterizzato dal rifiuto, segue poi un percorso di adattamento a questa scelta anti-convenzionale che richiede un impegno congiunto dei genitori e dei partner della nuova coppia. Il processo di legittimazione della scelta e la conseguente accettazione del partner straniero ha delle ripercussioni positive sul funzionamento della coppia e sul senso di autostima dei partner (Petit, 2001, in Gozzoli e Regalia, 2005; LorenzoBlanco, Bares e Delva, 2013). La vicinanza fisica ai genitori gioca, in tale processo, un ruolo fondamentale. Il coniuge che vive nella propria terra d'origine ha maggiore possibilità di contare sul sostegno della famiglia. In questi casi si viene a creare uno sbilanciamento relazionale tra i partner (Yuh, 2002, in Gozzoli e Regalia, 2005); tutto ciò può portare lo "straniero" ad essere inserito nella famiglia del coniuge a patto che abbandoni la propria cultura d'origine per adottare quella del paese in cui ora risiede. Il coniuge straniero può cedere o attuare una forma di resistenza passiva, mantenendo comunque attiva la propria identità culturale, apparentemente sopraffatta da quella del partner. 


\subsection{La formazione della coppia}

Le coppie caratterizzate da una diversità culturale devono negoziare le loro visioni del mondo per poter costruire una propria identità familiare, un fare comune e condiviso a partire dagli aspetti più semplici della vita quotidiana (Balsamo, 2003). Il raggiungimento di un'intesa di coppia positiva dipende dalla capacità dei coniugi di riconoscere le differenze, di prendersi cura del legame di coppia e dalla presa di coscienza dell'originaria e irriducibile differenza che caratterizza qualsiasi unione coniugale (Scabini e Cigoli, 2000). Molte coppie miste riferiscono che nelle prime fasi dell'innamoramento vengono esaltati gli aspetti di somiglianza con il partner, risultati fondamentali per lo sviluppo di un interesse reciproco e per la successiva nascita della relazione amorosa (Lewis, Yancey e Bletzer, 1997; Wieling, 2003). Inizialmente la paura della diversità, vissuta come minacciosa, può determinare una negazione della stessa e un riconoscimento dell'altro come simile a sé. Vi è poi la tendenza all'idealizzazione del partner, funzionale al consolidamento del legame e alla qualità della scelta fatta. Nella fase successiva emergono gli aspetti precedentemente ai margini della rappresentazione nella vita di coppia, mettendone in discussione il funzionamento abituale (Molina, Estrada e Burnett, 2004); la crisi può essere innescata da svariati fattori: differenze di status sociale, aspettative legate al genere, legami con le famiglie d'origine.

La consapevolezza delle reciproche differenze culturali impone dunque ai partner di attuare un lavoro di ristrutturazione del rapporto, che altrimenti può andare incontro ad una sofferenza non più gestibile (Gozzoli e Regalia, 2005); la difficoltà è ulteriormente determinata dalla non condivisione dello stesso universo culturale, che implica l'uso di strategie di risoluzione del conflitto differenti che rischiano di incrementare le distanze (Bratter e Eschbach, 2006).

Tuttavia, gli studi che hanno messo a confronto le coppie miste con quelle monoculturali non hanno rilevato differenze significative per il loro funzionamento. Nello specifico, non sono state riscontrate importanti discrepanze per quanto riguarda la stima di sé e la qualità della relazione (Gurung e Duong, 1999), al grado di soddisfazione ed espressione emotiva (Negy e Snyder, 2000). Inoltre, sembra che tali coppie presentino uno stile di attaccamento sicuro e un adattamento reciproco al comportamento negativo, scambiando reciprocamente rispetto e affetto (Gaines e Agnew, 2003, in Troy e Lewis-Smith, 2006) e una soddisfazione relazionale significativamente più alta rispetto a quelle intrarazziali (Troy e Lewis-Smith, 2006). L'apprezzamento sincero delle differenze culturali, la formazione e il mantenimento di una cultura di coppia e la disponibilità cognitiva ed emotiva alla crescita di ciascun partner, sono considerati fattori fondamentali per la 
soddisfazione delle coppie interculturali (Gaines e Brennan, 2001 cit. in Lombardi e Ardone, 2008).

\subsection{La costruzione dell 'identità di coppia}

La negazione delle differenze culturali tra i coniugi costituisce una minaccia alla stabilità del legame. Quando i partner iniziano ad avvertire la loro reciproca alterità, si viene ad innescare una crisi che impone loro di attuare un lavoro di ristrutturazione del legame nel quale si inserisce quel processo definito da Falicov (1995, in Gozzoli e Regalia, 2005) «transizione culturale» (p. 178) che consente alla coppia di costruire una propria identità. Secondo Gozzoli e Regalia (2005), prendersi cura delle radici culturali della coppia si traduce in un accompagnamento del partner nel proprio mondo culturale, in modo da proteggerlo da eventuali incomprensioni e da elementi minacciosi provenienti dal contesto sociale di appartenenza, nel riconoscimento e valorizzazione della cultura dell'altro e nel dare un equilibrato valore alle reciproche differenze.

Tra gli elementi maggiormente significativi nel funzionamento della coppia vi è la capacità di gestione del conflitto. A tal proposito, Corijn, Liefbroer e Gierveld (1996) ritengono che le unioni monoculturali, diversamente da quelle interculturali, condividano maggiormente esperienze sociali simili, fondamentali nel promuovere l'accordo coniugale. Di conseguenza, in base ai modelli di omogamia, le coppie miste mancano di questo processo primario di facilitazione dell'accordo coniugale. Gozzoli e Regalia (2005) ritengono che nelle coppie miste la possibilità di gestire adeguatamente le situazioni conflittuali è altamente correlata con il «grado di tolleranza e di flessibilità che ciascun partner riesce a mostrare a sé e all'altro» (p. 182). Le strategie impiegate sono spesso condizionate dal grado di coinvolgimento ed identificazione alla propria cultura o a quella del partner: nel caso in cui i partner ritengono la propria cultura un valore importante da preservare verranno favoriti i processi di confronto, scambio e negoziazione.

\subsection{La transizione alla genitorialità}

Per molte coppie miste la transizione alla genitorialità consente di acquisire consapevolezza sulla complessità della relazione interculturale (Gozzoli e Regalia, 2005). La nascita del primo figlio potrebbe divenire un "catalizzatore del conflitto" per quelle coppie interculturali che precedentemente abbiano minimizzato l'impatto delle differenze culturali (Tseng e 
Hsu, 1991, in Crippen e Brew, 2007; Romano, 2001, in Crippen e Brew, 2007). La transizione alla genitorialità sollecita, inoltre, questioni che vanno al di là delle dinamiche di coppia, ad esempio paure o competizioni tra le famiglie di origine in merito all'"accaparramento" del bambino a cui poter tramandare il proprio bagaglio culturale. È a partire dalla scelta del nome da dare al bambino che la coppia affronta in maniera esplicita ciò che intende salvaguardare della propria cultura d'origine; tale scelta è l'espressione del compromesso culturale che i coniugi hanno raggiunto. Questa fase coinvolge anche le rispettive famiglie d'origine: i nonni vedono nel nipote una garanzia della continuità identitaria (Tognetti Bordogna, 2004). Per ovviare al conflitto a volte si opta per un nome universale e riconosciuto in entrambe le culture; un nome che sia estraneo alle culture di origine dei genitori o ancora uno per ogni cultura.

Altro aspetto critico è relativo all'educazione religiosa. Spesso la coppia non affronta adeguatamente le questioni relative all'appartenenza religiosa, poiché sono quelle meno disponibili al compromesso. L'assenza di un'adeguata negoziazione religiosa tra i partner potrà avere delle ripercussioni negative nel momento in cui i partner cercheranno di trasmettere al figlio il patrimonio valoriale e normativo delle rispettive culture di appartenenza, spinti dalla voglia di mantenere vivi precisi riti e pratiche religiose, connotate da una forte valenza simbolica. Alcune coppie decidono di perseguire la strada del compromesso e di insegnare i principi fondamentali di entrambe le religioni in nome dell'universalismo religioso, altre preferiscono che il figlio sia completamente assimilato al credo della società di accoglienza (Tognetti Bordogna, 2004).

Infine, un'altra questione pregnante riguarda l'insegnamento della lingua. L'ipotesi del bilinguismo è quella maggiormente perseguita, considerata un'opportunità, in termini di adattamento e successo (Gaspar, 2009). Vi sono coppie che, invece, scartano la possibilità di insegnare più lingue ai propri bambini, temendo che questo possa produrre un certo grado di ambivalenza identitaria. Vi sono genitori, infine, che ricorrono all'uso della lingua di origine per comunicare al figlio emozioni e per creare una certa intimità familiare (Burk, 2004), utilizzando invece l'altro idioma per le comunicazioni "sociali".

\section{Ostacoli e criticità nel percorso evolutivo della coppia mista}

Come si è visto, la riflessione scientifica sulle coppie miste è di ampio respiro, i ricercatori infatti si sono concentrati non solo sull'analisi delle questioni prettamente psicologiche inerenti la formazione di tali unioni, ma 
anche sulla vulnerabilità delle stesse; si tratta di una potenziale fragilità causata dai vincoli religiosi, culturali e giuridici che, a volte, allontanano le coppie interraziali dall'essere un laboratorio relazionale privilegiato, così come spesso vengono definite in ambito psicosociale (Fruggeri, 2005).

Un aspetto di grande rilevanza è il rapporto con il mondo esterno. Oltre alla famiglia di origine, anche la rete amicale e le comunità di appartenenza possono ostacolare/facilitare la relazione mista. A tali coppie viene, spesso, a mancare il supporto sociale, fondamentale per la serenità e la durata del rapporto (Herman e Campbell, 2012). Le coppie interetniche possono incontrare comunità "chiuse" nei confronti della diversità e ciò determina un'esclusione formale o informale della coppia (Fenaroli e Panari, 2006). La stessa società occidentale condivide ancora in larga misura stereotipi negativi nei confronti di membri che intraprendono una relazione mista (Root, 1996, in Lombardi e Ardone, 2008; Gaines e Ickes, 1997, in Lombardi e Ardone 2008), vista non come unione romantica tra due individui, ma come unione tra due gruppi sociali diversi ed in contrapposizione tra loro (Fenaroli e Panari, 2006).

Soffermandoci sul contesto italiano, lo studio condotto da Lombardi e Ardone (2008) evidenzia che i partner italiani vengono accettati più frequentemente rispetto a quelli stranieri nei rispettivi contesti sociali. Laddove i partner stranieri trovano difficoltà di inserimento appaiono più propensi a svalutare il paese del partner, a giudicare negativamente alcuni comportamenti, abitudini e caratteristiche dei connazionali e, nello stesso tempo, a difendere il proprio paese di origine e la propria identità etnica.

Dallo studio condotto da Killian (2001) emerge che le reazioni negative del contesto sociale vengono percepite maggiormente dal partner appartenente al gruppo minoritario rispetto al partner del gruppo di maggioranza; allo stesso tempo, però, gli ostacoli esterni possono rivelarsi una risorsa: alcuni membri delle coppie miste possono infatti reagire alle pressioni negative delle famiglie e della società intensificando il livello di coesione tra $\mathrm{i}$ partner (Luke, 2003; Seshadri e Knudson-Martin, 2013).

Alcuni autori si sono interrogati sugli elementi di stabilità o di fragilità presenti in queste unioni (Bratter e King, 2008; Wong, 2009), le cui motivazioni sono rintracciabili non solo nelle possibili tensioni interne ma anche in quelle esterne alla coppia. Le prime sono attribuibili alle differenze culturali e religiose dei due partner, ai differenti copioni sessuali, ma anche al divario del livello di istruzione e di età; quest'ultima, in particolare, riscontrabile nella tipologia marito italiano (38 anni in media) e moglie straniera (31 anni di media) (Istat, 2012). Le tensioni esterne sono, invece, causate dallo stigma e dal pregiudizio sociale che appaiono essere fortemente dipendenti dalla combinazione razza/genere dei partner. 
Lewandowski e Jackson (2001), in tal senso, ricordano che gli uomini americani hanno ostacolato per lungo tempo l'unione tra le "loro" donne e gli uomini africani, a causa della competizione sessuale e, soprattutto, dell'intenzione di vantare una supremazia etnica. La tipologia di coppie, riconducibile entro la dicotomia bianco/nero, ad esempio, era percepita come l'unione interraziale più ripugnante, poiché in essa era rilevabile la violazione, da parte dell'uomo soprattutto, delle norme sociali relative ai modi riconosciuti come più appropriati per formare una coppia. La tendenza sociale, in genere, era quella di percepire l'uomo sposato con una donna straniera come penalizzato, anche professionalmente, a causa dell'assenza di un'identità razziale, contrariamente a coloro i quali contraevano un matrimonio endogamo (ibidem).

La spiegazione di tale posizione, a parere di Lewis e Ford-Robertson (2010), può essere rintracciata nella teoria relativa al processo di assimilazione di Gordon (1991), vale a dire nella tendenza della classe mediabianca-americana a percepire i gruppi sociali minoritari, piuttosto che una fonte di arricchimento per il paese di accoglienza, come assimilabili. Il risultato che ne è conseguito, dunque, è riscontrabile nel percorso del processo di assimilazione che, in casi come questo presenti in molti paesi, ha preteso l'abbandono da parte dei gruppi minoritari dei principali aspetti delle loro culture e il relativo riconoscimento di uno spazio esiguo ai valori peculiari delle stesse. Negli anni gli americani hanno comunque avuto l'opportunità di entrare in contatto con numerose etnie e, di conseguenza, hanno potuto ridurre lo stereotipo e stabilire nuove amicizie, come ad esempio i più giovani all'interno del college (Qian, 1997).

Il vecchio antagonismo ha ceduto, in tal modo, il passo ai matrimoni misti.

Anche la motivazione che sta alla base dell'evento migratorio sembra influenzare la percezione sociale relativa alla coppia mista; può trattarsi di migrazione dettata da motivi politici, da ricongiungimento familiare, da esigenze lavorative o di studio o, appunto, causate dalla formazione della coppia; tutti motivi, comunque, che in vari modi impongono ai due partner il confronto con gli ostacoli, e le richieste, poste dal sociale. A questi la coppia può rispondere in maniera più o meno adattiva, anche alla luce del sostegno sociale, percepito e ricevuto, al quale le famiglie birazziali fanno riferimento. Il supporto offerto dalla famiglia d'origine, nello specifico, gioca un ruolo strategico poiché contribuisce ad agevolare la legittimità dello status sociale di coppia, così come avviene per le famiglie ricostituite (Cherlin, 1978; Lewis e Ford-Robertson, 2010). La rete familiare e la rete amicale possono incrementare le possibilità di successo della coppia, incoraggiando a valorizzare le differenze dei due partner e a sostenere il continuo confronto richiesto dall'unione mista. 
A causa della lontananza geografica, in taluni casi, il partner straniero giova del supporto offerto dalla famiglia di origine e dalle amicizie del partner autoctono; un dato, questo, che impone la riflessione sulla posizione della famiglia dell'immigrato, che sembra dover fare i conti con la scelta biculturale (Novara, Romano e Petralia, 2011).

Alcune ricerche hanno evidenziato, a tal proposito, che le famiglie di origine sono le prime ad opporsi all'unione mista oppure aumentano le interferenze in occasione della nascita del primo figlio della coppia, sentendosi in dovere di dare dei suggerimenti sulle scelte educative finalizzate alla continuità dei valori della propria appartenenza etnica (Spickard, 1989). Il rifiuto da parte della famiglia allargata di una possibile biculturalità, che può esprimersi anche nella scelta del nome da attribuire al nascituro o dell'educazione religiosa, quindi, può produrre o acuire il conflitto fra i partner o persino incoraggiare, questi ultimi, all'isolamento sociale o a cercare la compagnia di altre famiglie miste (Tognetti Bordogna, 2004).

A queste può aggiungersi il ruolo del contesto sociale, a volte ostico e poco supportivo nei confronti della mixità, poiché incapace di rispondere in modo adeguato alle esigenze di queste famiglie che, nonostante la numerosità $\left(59,2 \%\right.$ nell'anno 2001 nei Paesi a Sviluppo Avanzato $\left.{ }^{1}\right)$, sembrano rappresentare un'eccezione a causa della tendenza generale all'endogamia (Allievi, 2006). Il funzionamento di queste famiglie, in fondo, racconta anche delle società e delle comunità che le accolgono, del loro funzionamento più o meno efficace, dei valori e dei disvalori. Gli adattamenti derivano da scontri, incroci o innesti (Di Vita e Granatella, 2009).

La coppia può reagire a tali tensioni, dunque, diminuendo la dissonanza percepita attraverso l'accettazione e la compatibilità delle differenze e, ancora, incrementando la realizzazione delle aspettative; la negoziazione delle proprie peculiarità culturali e la costante ricerca di nuovi equilibri, tra identità etnica ascritta e identità etnica acquisita, pongono le basi per la costruzione di un'identità familiare.

Il numero di separazioni ${ }^{2}$, coniugali e more uxorio, delle coppie interraziali suggerisce che tali obiettivi non sempre sono raggiunti, motivo per il quale siamo esortati a riflettere sull'importanza dell'assenza di sostegno sociale che caratterizza queste esperienze familiari che, purtroppo, come nel

${ }^{1}$ Cfr. Maffioli D. e Paterno A. (2008), Famiglie straniere e coppie miste. Immagini dai censimenti 1991 e 2001. Annali del Dipartimento di Scienze Statistiche dell'Università degli Studi di Bari, 7, pp. 125-154, testo disponibile al sito: http://www.dip-statistica.uniba.it/ html/annali/2008/home2008.htm.

${ }^{2} \mathrm{Nel} 2005$ sono state pronunciate nei tribunali italiani 7.536 separazioni riguardanti “coppie miste" di coniugi, contro 4.266 concesse nell'anno 2000, con un incremento pari al $76,7 \%$ (Istat, 2010). 
caso di una separazione conflittuale, possano sfociare nella sottrazione internazionale di minori (Troy, Lewis-Smith e Laurenceau, 2006).

\section{La seconda generazione tra assimilazione e appartenenza etnica}

I figli delle coppie miste, aventi genitori biologici con diverse origini etniche, rappresentano l'espletamento della quarta fase del processo di assimilazione di Gordon (1991) (identificational assimilation), quella cioè in cui si registra l'accettazione sociale delle coppie interraziali.

Questa seconda generazione, in virtù dell'appartenenza a famiglie biculturali, ha la possibilità di riflettere entro una prospettiva multirazziale, di possedere migliori capacità relazionali, e dimostra anche sensibilità e vulnerabilità dinanzi ai diversi atteggiamenti dei genitori, alle reazioni del gruppo dei pari e alle attenzioni ambivalenti ricevute a scuola o all'interno della più ampia comunità. Gli stessi hanno anche la possibilità di decidere con quale delle due etnie genitoriali identificarsi, anche se la scelta per una precisa categoria etnica può essere difficoltosa a causa dell'influenza delle aspettative, e delle norme sociali, parzialmente trasmesse al figlio dai genitori; ancora, questi minori possono optare per un'identificazione autonoma e indirizzata verso comportamenti e tradizioni culturali lontane dal background della famiglia (Rosenblatt, Karis e Powell, 1995 cit. in Bratter e Eschbach, 2006; Miano e Salerno, 2010).

La flessibilità mentale e le possibili risorse, interne ed esterne, che vengono offerte a questi minori, però, vengono negate agli stessi in caso di separazione dei genitori, così come spesso accade in occasione di separazioni coniugali conflittuali endogame. I figli, infatti, possono essere loro malgrado coinvolti nei conflitti distruttivi dei genitori e non rari sono gli eventi di cronaca relativi alla sottrazione internazionale di minori. Questi ultimi, ad esempio, possono essere portati da un genitore - senza il consenso dell'altro che viene anche privato del diritto alla potestà genitoriale - in un paese diverso da quello di residenza (legal kidnapping o custodial interference). La motivazione che esorta un genitore a compiere tale rapimento, in genere, è quella della speranza di ottenere l'affidamento della prole, contrariamente a quanto deciso dal foro del paese di residenza o, ancora, per agire violenza nei riguardi dell'ex compagno/a, ma anche dei figli che vengono repentinamente sradicati dalle loro abitudini.

Le leggi dei diversi Stati, atte a combattere tale tipo di comportamento, sono diverse. In alcuni Paesi tali rapimenti sono classificati come reati o crimini, imponendo conseguenze penali, anche alla luce della possibilità che i tribunali possano individuare, ad esempio, prove oggettive di violenza 
domestica, intesa come una delle ragioni per le quali il minore è stato sottratto dalla custodia di un genitore (rapimento protettivo). In altri Paesi, invece, l'indagine non è sempre avviata immediatamente, motivo per il quale il più grave e dirompente dei rapimenti può avere meno probabilità di ricevere l'applicazione tempestiva della legge.

L'Italia, per contrastare tale fenomeno, ha sottoscritto la Convenzione dell'Aja sugli Aspetti civili della sottrazione internazionale di minori del 1980 e la Convenzione del Lussemburgo sul Riconoscimento e l'esecuzione delle decisioni in materia di affidamento dei minori e di ristabilire l'affidamento (1980), istituendo presso il Ministero di Grazia e Giustizia l'Autorità Centrale. La tutela del minore, però, è ostacolata non solo dall'obbligatoria assistenza legale, che richiede un notevole impegno economico alle parti, ma anche da una sorta di difesa ad oltranza del proprio connazionale da parte del paese partner assicurando, allo stesso, l'impunità $^{3}$. Il diritto di tutela dell'infanzia è stato anche oggetto della Convenzione Internazionale sui diritti del fanciullo (NY, 1989) anch'essa in difesa del diritto dei minori a non subire spostamenti o ritorni illeciti all'estero (art. 11).

La tutela del minore, e delle relazioni genitoriali, è anche oggetto di una legge federale americana - l'International Parental Kidnapping Crime Act (IPKCA) - la quale sottolinea l'importanza dell'emanazione di uno statuto uniforme da parte di ogni singolo Stato, al fine di privare i rapitori di rifugi sicuri, di eliminare gli ostacoli alla cooperazione fra i funzionari di polizia nel localizzare i bambini trasferiti illecitamente e di aiutare i Procuratori a portare i rapitori alla giustizia. Una rete interstatale di agenti delle forze dell'ordine e di pubblici ministeri impegnati ad applicare la stessa legge sulla sottrazione internazionale di minori da parte dei genitori, inoltre, potrebbe avere un notevole effetto educativo sugli aspiranti rapitori.

\section{Riferimenti bibliografici}

Allievi S. (2006). Cosa vuol dire "coppie miste"? Il vissuto e le interpretazioni. In: Zilio-Grandi I., a cura di, Sposare l'altro. Matrimoni e matrimoni misti nell'ordinamento italiano e nel diritto islamico. Venezia: Marsilio, pp. 11-41.

Balsamo F. (2003). Famiglie di migranti: trasformazioni dei ruoli e mediazioni culturali. Roma: Carocci.

${ }^{3}$ Cfr. Gruppo di lavoro per la Convenzione dell'infanzia e dell'adolescenza (Gruppo CRC) (2009). I diritti dell'infanzia e dell'adolescenza in Italia. Secondo Rapporto Supplementare alle Nazioni Unite sul monitoraggio della Convenzione sui diritti dell'infanzia e dell'adolescenza in Italia, novembre 2009. 
Bratter J.L. e Eschbach K. (2006). "What about the couple?” Interracial marriage and psychological distress. Social Science Research, 35, 1025-1047. DOI: 10.1016/j.ssresearch.2005.09.001

Bratter J.L. e King R.B. (2008). "But Will It Last?”: Marital Instability Among Interracial and Same-Race Couples. Family Relations, 57, 2, 160-171. DOI: 10. $1111 / \mathrm{j} .1741-3729.2008 .00491$

Burk C. (2004). Living in Several Languages: Implications for Therapy. Journal of Family Therapy, 26, 314-39. DOI: 10.1111/j.1467-6427.2004.00287.x

Carrà E. (2011). Famiglie fragili. In: Bramanti D. e Carrà E., a cura di, Buone pratiche nei servizi alla famiglia. Famiglie fragili e famiglie con anziani non autosufficienti. Roma: Osservatorio Nazionale sulla Famiglia, pp. 45-212.

Cherlin A. (1978). Remarriage as an Incomplete Institution. American Journal of Sociology, 84, 634-650. DOI: 10.1086/226830

Corijn M., Liefbroer A.C. e Gierveld J. (1996). It takes two to tango, doesn't it? The influence of couple characteristics on the timing and birth of the first child. Journal of Marriage and the Family, 58, 117-126. DOI: 10.2307/353381

Crippen C. e Brew L. (2007). Intercultural Parenting and the Transcultural Family: A Literature Review. The Family Journal, 15, 107-115. DOI: 10.1177/ 1066480706297783

Di Vita A.M. e Granatella V., a cura di (2009). Famiglie in viaggio. Narrazioni di identità migranti. Roma: Magi.

Fenaroli P. e Panari C. (2006). Famiglie miste e identità culturali. Roma: Carocci.

Fruggeri L. (2005). Diverse normalità. Psicologia sociale delle relazioni familiari. Roma: Carocci.

Gaspar S. (2009). Mixed marriages between European free movers. CIES e Working Paper, 65, CIES-ISCTE, Lisbon, testo disponibile al sito: www.cies.iscte.pt/ destaques/documents/CIES-WP65_Gaspar.pdf

Gordon M.M. (1991). Assimilation in American life: The role of race, religion, and national origins. In: Yetman N.R., Majority and minority: The dynamics of race and ethnicity in American life. Boston: Allyn Bacon, pp. 272-283.

Gozzoli C. e Regalia C. (2005). La coppia mista. In: Gozzoli C. e Regalia C., Migrazioni e famiglie. Percorsi, legami e interventi psicosociali. Bologna: il Mulino, pp. 153-206.

Gurung A.R. e Duong T. (1999). Mixing and matching: assessing the concomitant of mixed-ethnic relationships. Journal of Social and Personal Relationships, 16, 5, 639-657. DOI: 10.1177/0265407599165005

Herman M.R. e Campbell M.E. (2012). I wouldn't, but I can. Attitudes toward interracial relationships. Social Science Research, 41, 343-358. DOI: 10.1016/ j.ssresearch.2011.11.007

Istat (2012), Separazioni e divorzi in Italia, testo disponibile al sito: http://www. istat.it/it/archivio/66665

Killian K.D. (2001). Reconstituting racial histories and identities: the narratives of interracial couples. Journal of Marital and Family Therapy, 27, 1, 27-42. DOI: $10.1111 / \mathrm{j} .1752-0606.2001 . t b 01137$

Lewandowski D.A. e Jackson L.A. (2001). Perceptions of Interracial Couples: 
Prejudice at the Dyadic Level. Journal of Black Psychology, 27, 288-303. DOI: 10.1177/0095798401027003003

Lewis R. e Ford-Robertson J. (2010). Understanding the Occurrence of Interracial Marriage in the United States Through Differential Assimilation. Journal of Black Studies, 41, 405-420, testo disponibile al sito: http://www.jstor.org/stable 12784894

Lewis R., Yancey G. e Bletzer S.S. (1997). Racial and nonracial factors that influence spouse choice in black/white marriages. Journal of Black Studies, 28, 60-78.

Lombardi M. e Ardone R. (2008). Le coppie miste tra risorse e difficoltà. Lavoro presentato alle Giornate di studio della Sezione di Psicologia Sociale dell'AIP Spazi interculturali: trame, percorsi, incontri, Roma, 18-19 settembre.

Lorenzo-Blanco E.I., Bares C.B. e Delva J. (2013). Parenting, family processes, relationships, and parental support in multiracial and multiethnic families: an exploratory study of youth perceptions. Family relations, 62, 125-139. DOI: $10.1111 /$ j.1741-3729.2012.00751

Luke C. (2003). Global Mobilities. Crafting Identities in Interracial Family. International Journal of Cultural Studies, 6, 4, 379-401. DOI: 10.1177/ 136787790364001

Miano P. e Salerno A. (2010). Migrazione e adolescenza: psicodinamica di una doppia transizione, Convegno Nazionale La relazione tra famiglia e scuola: adolescenza, disabilità, differenze, Ferrara, 7 maggio.

Molina B., Estrada D. e Burnett, J.A. (2004). Cultural Communities: Challenges and Opportunities in the Creation of "Happily Ever After" Stories of Intercultural Couplehood. The Family Journal: Counseling And Therapy For Couples And Families, 2, 139-147. DOI: 10.1177/1066480703261962

Negy C. e Snyder D.K. (2000). Relationship satisfaction of Mexican American and non Hispanic White American interracial couples: Issues of acculturation and clinical intervention. Journal of Marital and Family Therapy, 26, 293-304. DOI: $10.1111 / \mathrm{j} .1752-0606.2000 . t b 00299$

Novara C., Romano F. e Petralia V. (2011). Famiglie biculturali: la soddisfazione familiare tra reti di supporto e comunità. In: Granatella V., a cura di, Reciproci sguardi. Sistemi migranti e costruzione intersoggettiva di pratiche e saperi. Milano: Franco Angeli, cap. 7, pp. 156-180.

Qian Z. (1997). Breaking the Racial Barriers: Variations in Interracial Marriage between 1980 and 1990. Demography, 34, 478-500. DOI: 10.2307/2061704

Salerno A. (2010). Relazioni interculturali e omosessuali tra pregiudizi e risorse. In: Salerno A. Vivere insieme. Tendenze e trasformazioni della coppia moderna. Bologna: il Mulino, cap. 4, pp. 159-192.

Scabini E. (1985), a cura di, L'organizzazione famiglia tra crisi e sviluppo. Milano: Franco Angeli.

Scabini E. e Cigoli V. (2000). Il famigliare. Legami, simboli e transizioni. Milano: Raffaello Cortina.

Scabini E., Regalia C. e Giuliani C. (2007). La famiglia nell'incontro con le culture. In: Mazzara B. (Ed.), Prospettive di psicologia culturale. Modelli teorici e contesti d'azione. Roma: Carocci. 
Seshadri G. e Knudson-Martin (2013). How couples manage interracial and intercultural differences: implications for clinical practice. Journal of Marital \& Family Therapy, 39, 1, 45-58. DOI: 10.1111/j.1752-0606.2011.00262

Spickard P.R. (1989). Mixed blood: Intermarriage and ethnic identity in twentiethcentury America. Madison: The University of Wisconsin Press.

Tognetti Bordogna M. (2004). L'incremento dei matrimoni misti. Verso una società transculturale. In: Tognetti Bordogna M. e Seidita M. Le famiglie miste. Laboratori culturali nella società trentina. Trento: Provincia Autonoma.

Troy A.B., Lewis-Smith J. e Laurenceau J.P. (2006). Interracial and intraracial romantic relationships: the search for differences in satisfaction, conflict and attachment style. Journal of Social and Personal Relationships, 23, 1, 65-80. DOI: $10.1177 / 0265407506060178$

Wieling E. (2003). Latino/a and white marriages: A pilot study investigating the experiences of interethnic couples in the United States. Journal of Couple and Relationship Therapy, 2, 41-55. DOI: 10.1300/J398v02n02_04

Wong M.K.B.G. (2009). Strengthening connections in interracial marriages through pre-marital inventories: a critical literature review. Contemporary Family Therapy, 31, 251-261. DOI: 10.1007/s10591-009-9099-1 